\title{
Lateral hopping conductivity and large negative magnetoresistance in InAs/AIGaAs self-organized quantum dots
}

\author{
B Kochman, S Ghosh, J Singh and P Bhattacharya \\ Solid State Electronics Laboratory, Department of Electrical Engineering and \\ Computer Science, University of Michigan, Ann Arbor, MI 48109-2122, USA
}

Received 12 April 2002

Published 16 July 2002

Online at stacks.iop.org/JPhysD/35/L65

\begin{abstract}
We report experimental studies on lateral transport in self-organized quantum dots. We find that below $100 \mathrm{~K}$, conduction occurs through interdot hopping and that experimental results are described quite well by a variable-range hopping model. In the hopping regime, the in-plane conductance varies as $G=G_{0} \exp \left[\left(-T_{0} / T\right)^{1 / 3}\right]$, and $T_{0}$ is found to be $7100-9400 \mathrm{~K}$. We have also observed a large negative magnetoresistance in this structure.
\end{abstract}

Electronic phenomena in self-organized quantum dots have interested researchers for quite some time [1-6]. In particular, lateral transport in structures including these dot layers has attracted significant attention [7-10]. The self-organized dot system is highly suitable for studying conduction mechanisms in localized states in a quasi-zero-dimensional space. In figure 1(a), we show a schematic of the density of states in a system of self-organized dots. Theoretical and experimental results show that there are at least two states, shown as $E_{1}$ and $E_{2}$, where the electronic wavefunction is substantially localized within each dot and there are extended states starting at $E_{\mathrm{C}}$, where the electronic wavefunction is spread over the entire sample $[3,4]$. Due to the size fluctuations in the dots, the localized states have a certain spread over energy (about $30-50 \mathrm{meV}$ ) as shown in figure $1(a)$.

From the description given above we can expect several interesting regimes of transport in the lateral plane of the dots:

(a) At low temperature and very low electron densities, only the ground state of the dots is occupied. Since the electrons are highly localized in the ground state, we expect negligible conductivity.

(b) At low temperature and moderate electron densities, the excited state is occupied but the extended states are not. The electron wavefunction of the excited states is sufficiently spread to allow significant coupling between (a)

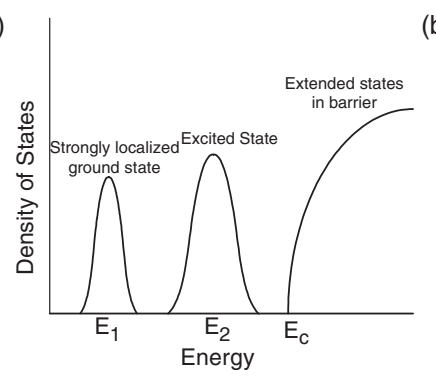

(b)

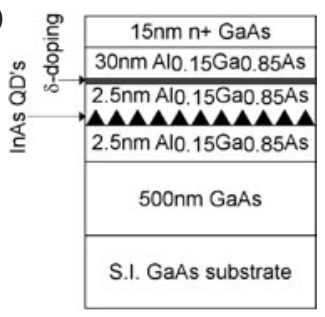

(c)

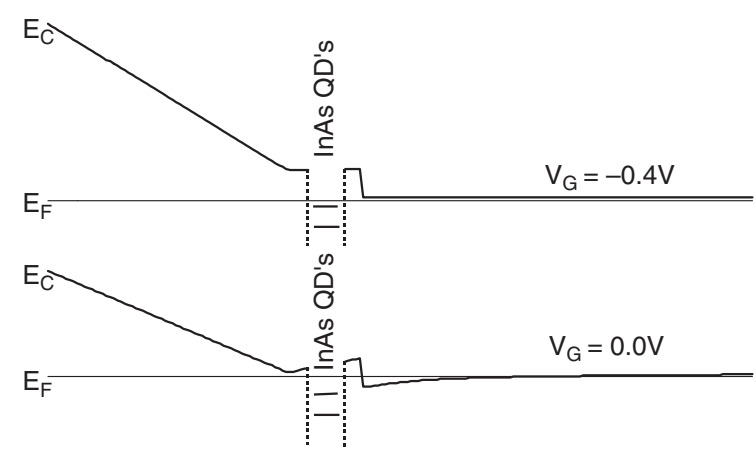

Figure 1. (a) A schematic of the density of states in self-organized dots; (b) quantum dot heterostructure grown by MBE;

(c) conduction band diagram of the device under zero and reverse bias. 
dots. As in amorphous materials, we expect variablerange hopping where the probability of an electron hopping to a neighbouring site depends on the distance to that site and the difference in energy between that site and the starting energy. Such hopping is mediated by phonons.

(c) At higher temperatures than the case above, but at similar electron densities, we expect that electrons are thermally excited to the extended states and transport occurs primarily through the extended states.

(d) At high electron densities, the extended states are occupied. In this case, transport is essentially similar to transport in degenerate semiconductors.

In this paper, we examine all these regimes using a gated device. However, due to the its interesting nature, we focus our discussions on the regime of variable-range hopping.

We etched mesas in the shapes of bars into the heterostructure which were grown by molecular beam epitaxy (MBE) and are shown in figure $1(b)$. The quantum dots are approximately pyramidal with a base length of about $20 \mathrm{~nm}$, a height of $6-8 \mathrm{~nm}$, and a density of $10^{10}-10^{11} \mathrm{dots}_{\mathrm{cm}}^{-2}$, as observed by atomic force microscopy (AFM) and crosssection transmission electron microscopy (XTEM) [4]. We then evaporated and annealed $\mathrm{Ni} / \mathrm{Ge} / \mathrm{Au}$ contacts. To enable us to modulate the Fermi level and individually explore the various conduction regimes describe above, we also formed a Schottky gate by $\mathrm{Ni} / \mathrm{Au}$ evaporation. The device is $50 \mu \mathrm{m}$ long and $80 \mu \mathrm{m}$ wide.

To estimate electron densities, we performed a selfconsistent calculation of the one-dimensional Schroedinger and Poisson equations assuming two states (four electrons) per dot. The band diagrams, calculated at $50 \mathrm{~K}$, are shown in figure $1(c)$. At zero gate voltage, we find that we have a large density of carriers in the well, however by lowering the gate voltage to $-0.4 \mathrm{~V}$, we find that the Fermi level drops below the energy of the bottom of the well. At $V_{\mathrm{G}}=-0.4 \mathrm{~V}$ we calculate the electron density in the well to be $3 \times 10^{9} \mathrm{~cm}^{-2}$ and three electrons per dot.

The results of resistance measurements conducted on this device are shown in figure $2(a)$. As would be expected for either thermally activated conduction or hopping conduction, the resistance decreases with increasing temperature. In the former mechanism, without sufficient thermal energy to excite electrons above the bandgap discontinuity, most electrons are trapped in the confined dot states and therefore cannot contribute to conduction. In the latter conduction mechanism because phonons are required to conserve energy in during a hop, the higher phonon density at a higher temperature increases the hopping rate and thereby increases conductivity. Our data exhibit two distinct slope regions, which we attribute to these two conduction mechanisms. The breakpoint between the conduction regimes is at about $100 \mathrm{~K}$.

Above $100 \mathrm{~K}$, the dominant conduction mechanism is thermal activation, which is characterized by a temperaturedependent resistance:

$$
R_{\mathrm{s}}=R_{\mathrm{s} 0}(T) \exp \left(\frac{E_{\mathrm{C}}-E_{\mathrm{F}}}{k_{B} T}\right)
$$

The temperature dependence of $R_{\mathrm{s} 0}$ is due to the temperature dependence of resistivity in the $\mathrm{Al}_{0.15} \mathrm{Ga}_{0.85}$ As layer, which is
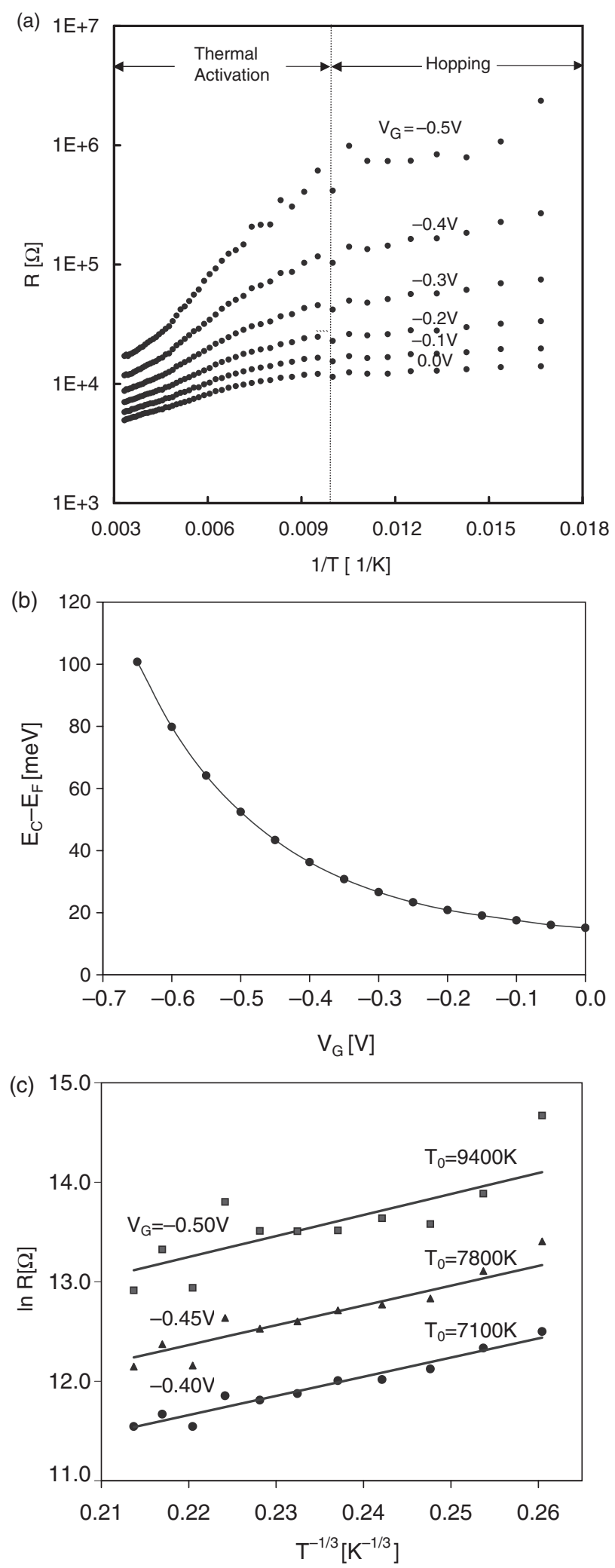

Figure 2. (a) Resistance of InAs quantum dot layer for several gate voltages. At high temperatures thermally activated conduction dominates, but at low temperatures hopping conduction dominates; (b) the Fermi level position in the dots extracted from the data; (c) measured and calculated resistance shown in a magnified temperature range within the hopping conduction regime. 
negligible compared to the exponential factor. The slope of the curves in this conduction region gives the position of the Fermi level, which is plotted in figure $2(b)$.

Below $100 \mathrm{~K}$ at sufficiently negative gate biases, the thermal energy of electrons is insufficient to overcome the activation barrier, and hopping conduction dominates. When $E_{\mathrm{C}}-E_{\mathrm{F}}>40 \mathrm{meV}$, electrons are primarily confined to the ground state where they are strongly localized. We are unable to measure the conductivity of electrons in these states since the current levels are comparable to the background noise current. This is expected since electrons are strongly localized in the ground state and there is negligible overlap between the electron wavefunctions of neighbouring dots.

For the case where $E_{\mathrm{C}}-E_{\mathrm{F}} \leqslant 40 \mathrm{meV}$, electrons reside in the excited state where the electron wavefunctions are less localized. Here, at low temperatures, we expect that Mott's model [11] of variable-range hopping conductivity may be applicable. The planar distribution of confined states leads to a $T^{-1 / 3}$ law $[12,13]$, resulting in a hopping rate of

$$
p=v_{\mathrm{ph}} \exp \left[\left(-\frac{T_{0}}{T}\right)^{1 / 3}\right],
$$

where

$$
T_{0}=\frac{27 \alpha^{2}}{\pi N\left(E_{\mathrm{F}}\right) k_{B}} .
$$

$\alpha$ is the decay rate of the quantum dot wavefunction (i.e. $\Psi \sim \exp (-\alpha R))$ and $v_{\mathrm{ph}}$ is the phonon frequency. The conductance is directly proportional to the hopping rate:

$$
\begin{aligned}
\frac{1}{R_{\mathrm{S}}} & =n_{\mathrm{s}} e \mu \approx \frac{1}{6} e^{2} p R^{2} N\left(E_{\mathrm{F}}\right) \\
& =\frac{1}{6} e^{2} R^{2} N\left(E_{\mathrm{F}}\right) v_{\mathrm{ph}} \exp \left[\left(-\frac{T_{0}}{T}\right)^{1 / 3}\right] .
\end{aligned}
$$

$R$ is the average hop distance. Figure 2(b) shows the hopping region magnified and plotted on a $T^{-1 / 3}$ axis, as is suggested by the model. We plot the results for $V_{\mathrm{G}} \leqslant-0.4 \mathrm{~V}$. Above this gate voltage, there is significant conduction in the well and extended states. The solid line is the fit to the data in accordance with equation (4) resulting in $T_{0}$ ranging from 7100 to $9400 \mathrm{~K}$. It should be noted, however, that the validity of the fit cannot be conclusively affirmed due to the limited range of the measured resistance values. Equation (3) allows us to estimate $\alpha$ if we can approximate the density of dot states, which is the ratio of the dot density $\left(\approx 10^{11} \mathrm{dots}^{-2}\right)$ to the photoluminescence line-width $(\approx 50 \mathrm{meV})$ yielding $N\left(E_{\mathrm{F}}\right) \approx 2 \times 10^{12} \mathrm{eV}^{-1} \mathrm{~cm}^{-2}$. Using the $T_{0}=8100 \mathrm{~K}$, the average value, we calculate $1 / \alpha \approx$ $250 \AA$, a reasonable value for the extent of an excited state wavefunction. At this gate voltage $(-0.4 \mathrm{~V})$, the Fermi level is about $40 \mathrm{meV}$ below the band-edge discontinuity according to equation (1), so electron occupation of the excited states is expected.

In materials where hopping is the mechanism responsible for conductivity, negative magnetoresistance (MR) has often been observed [14, 15]. Thus, we measured the MR of the selforganized quantum dot material to see if a similar phenomenon can be observed. The measurements were conducted with the magnetic field perpendicular to the sample surface. The MR of an ungated device, shown in figure 3(a), reveal a large
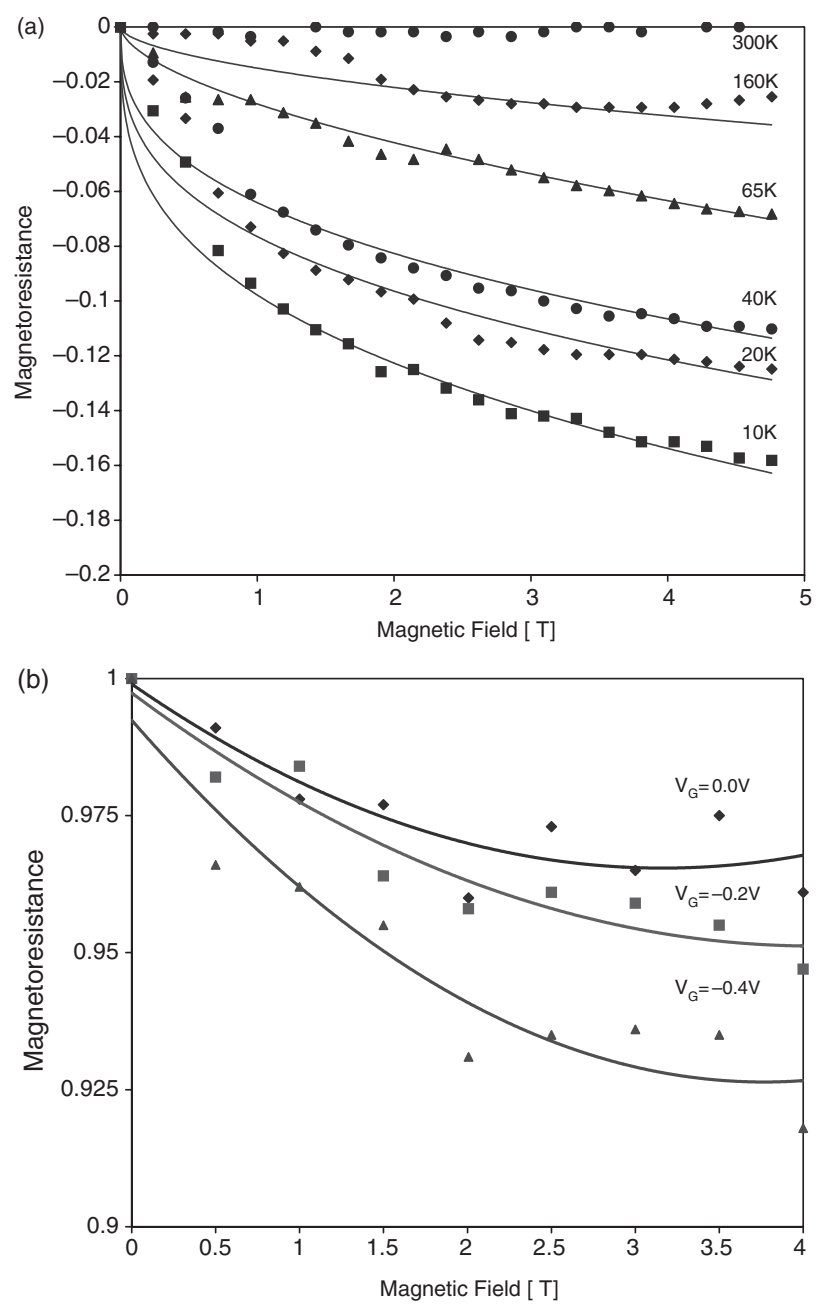

Figure 3. (a) MR measured on an ungated InAs quantum dot layer. Negative MR is observable below $160 \mathrm{~K}$ and increases in magnitude at lower temperatures. The data is fit with $\Delta R / R \propto B^{n} ;(b) \mathrm{MR}$ measured on a gated InAs quantum dot layer.

negative MR, observable at ambient temperatures as high as $160 \mathrm{~K}$. At lower temperatures, the negative MR becomes more pronounced. We find a field dependence of the MR: $\Delta R / R \propto B^{n}$ for fields greater than approximately $1 \mathrm{~T}$, but for lower fields the magnitude of the MR is smaller than would be expected from this relationship. The exponent ranges from $n=0.3$ at $10 \mathrm{~K}$ to $n=0.6$ at $65 \mathrm{~K}$. A power law has previously been found in amorphous $\mathrm{Ge}$ [16]. On the same heterostructure which was grown at a different time, we conducted a similar measurement using a gated device as shown in figure $3(b)$. As the Fermi level is driven deeper into the dots (i.e. $V_{\mathrm{G}}$ is made more negative), the magnitude of the negative MR increases. While the observation of a pronounced negative MR adds support to the assertion that conduction in the self-organized quantum dot system is via hopping, it should be noted that hopping is not the only phenomena which can explain it.

In conclusion, we studied lateral electron transport in self-organized quantum dot systems. At low temperatures, $T<100 \mathrm{~K}$, hopping conduction dominates while at higher temperatures, thermal activation of carriers is more prevalent. In the hopping regime, we use the Mott model fit to the observed data. The self-organized quantum dots also exhibit 
a large negative MR up to $160 \mathrm{~K}$ which further reinforces the hypothesis that hopping exists at a sufficiently low temperature.

\section{Acknowledgments}

This work is supported by the National Science Foundation under Grant ECS-9820129 and the Army Research Office (MURI program) under Grant DAAD-19-99-1-0198.

\section{References}

[1] Narihiro M, Yusa G, Nakamura Y, Noda T and Sakaki H 1997 Appl. Phys. Lett. $\mathbf{7 0} 105$

[2] Luyken R J, Lorke A, Govorov A O, Kotthaus J P Medeiros-Ribeiro G and Petroff P M 1999 Appl. Phys. Lett. 742486

[3] Ledentsov N N, Ustinov V M, Shchukin V A, Kopev P S, Alferov Zh I and Bimberg D 1998 Semiconductors 32343
[4] Bhattacharya P, Kamath K, Singh J, Klotzkin D, Phillips J, Jiang H-T, Chervela N, Norris T B, Sosnowski T, Laskar J and Murty M R 1999 IEEE Trans. Electron Devices 46871

[5] Susa G and Sakaki H 1997 J. Crystal Growth 175/176 730

[6] Martin G Y and Bastard G 1994 Solid State Commun. 92437

[7] Ribeiro E, Muller E, Heinzel T, Auderset H, Ensslin K, Medeiros-Ribeiro G and Petroff P M 1998 Phys. Rev. B 581506

[8] Sakaki H, Yusa G, Someya T, Ohno Y, Noda T, Akiyama H, Kadoya Y and Noge H 1995 Appl. Phys. Lett. 673444

[9] Horiguchi N, Futasugi T, Nakata Y and Yokoyama N 1997 Appl. Phys. Lett. 702294

[10] Phillips J, Kamath K, Brock T and Bhattacharya P 1998 Appl. Phys. Lett. 723509

[11] Mott N F 1969 Phil. Mag. 19835

[12] Pollitt S, Pepper M and Adkins C J 1976 Surf. Sci. 5879

[13] Hartstein A and Fowler A B 1976 Surf. Sci. 7319

[14] Mehra R M, Shyam R and Mathur P C 1983 Thin Solid Films 10081

[15] Hellman F, Tran M Q, Gebala A E, Wilcox E M and Dynes R C 1996 Phys. Rev. Lett. 774652

[16] Mell H and Stuke J 1970 J. Non-Cryst. Solids 4304 